\title{
Accurate Loss and Surface Mode Modeling in Fabricated Hollow-Core Photonic Bandgap Fibers
}

\author{
Eric Numkam Fokoua, Seyed Reza Sandoghchi, Yong Chen, Natalie. V. Wheeler, Naveen K. Baddela, John \\ R. Hayes, Marco N. Petrovich, David J. Richardson and Francesco Poletti \\ Optoelectronics Research Centre, University of Southampton, Highfield Campus, SO17 1BJ Southampton, UK \\ ernf1g10@orc.soton.ac.uk
}

\begin{abstract}
We present a method to reconstruct the cross-sectional profile of fabricated hollow-core photonic bandgap fibers from SEM images. For the first time, numerical simulations show a good agreement with measured loss and surface mode position.

OCIS codes: (060.4005) Microstructured fibers; (060.2280) Fiber design and fabrication
\end{abstract}

\section{Introduction}

Hollow-core photonic bandgap fibers (HC-PBGFs) have been proposed as a possible contender for the next generation of high capacity data transmission fibers. This class of waveguides offers a three-order of magnitude reduction in optical nonlinearity with respect to conventional silica fibers and near vacuum latency, but still suffers from relatively high loss values [1]. This loss, known to be fundamentally limited by scattering from surface roughness, is critically affected by fine structural details in the microstructured cladding, especially by those on the boundary between core and cladding.

The accurate numerical modeling and assessment of the properties of fabricated samples, an important process in the design of low-loss HC-PBGFs, has so far proved challenging. This crucial verification step is important to confirm whether or not a designed fiber operates as intended, to understand the effect of small structural imperfections and to provide feedback to the initial design. Traditionally, it has been carried out by modeling geometries obtained from scanning electron micrographs (SEM) of the fiber cross sections. The standard procedure consists of converting the grayscale SEM image into a two-level one and using edge detection routines to turn the boundaries of the air holes into smooth curves, usually splines $[2,3]$. The challenge arises because the limited resolution of the SEM images prevents one to simultaneously resolve the entire microstructured cross-section $(\sim 100 \mu \mathrm{m})$ and at the same time the smallest features such as the thin silica struts in the cladding (typically $\leq 200 \mathrm{~nm}$ ). Furthermore, the metallic coating required to prevent charging effects and the hard to control tilt of samples during the SEM acquisition contribute to exaggerating the value of the strut thickness, resulting in the images always showing a lower air-filling fraction (and therefore a photonic bandgap centered at longer wavelengths) than in practice [3]. Reported attempts to circumvent this problem have mainly consisted in increasing the magnification of the SEM images, thereby limiting the field of view to the core and to the first few rings surrounding it $[3,4]$. Although modal properties such as dispersion and birefringence can be reproduced, any reasonable comparison of measured and modeled loss values is foregone as only a portion of the fiber is simulated.

Here we propose a novel, fast and efficient method to faithfully reproduce HC-PBGF structures from SEM images. Because our method offers full control over the thickness of individual silica struts within the fiber crosssection, the resolution challenge is easily overcome. For the first time, simulations on the geometries of real HCPBGFs show a good agreement with measured fiber loss and surface modes position within the bandgap.

2. Reconstructing structural profile from SEMs

The procedure we use to reproduce the geometry profiles of HC-PBGFs from their SEM images is shown in Fig.1.
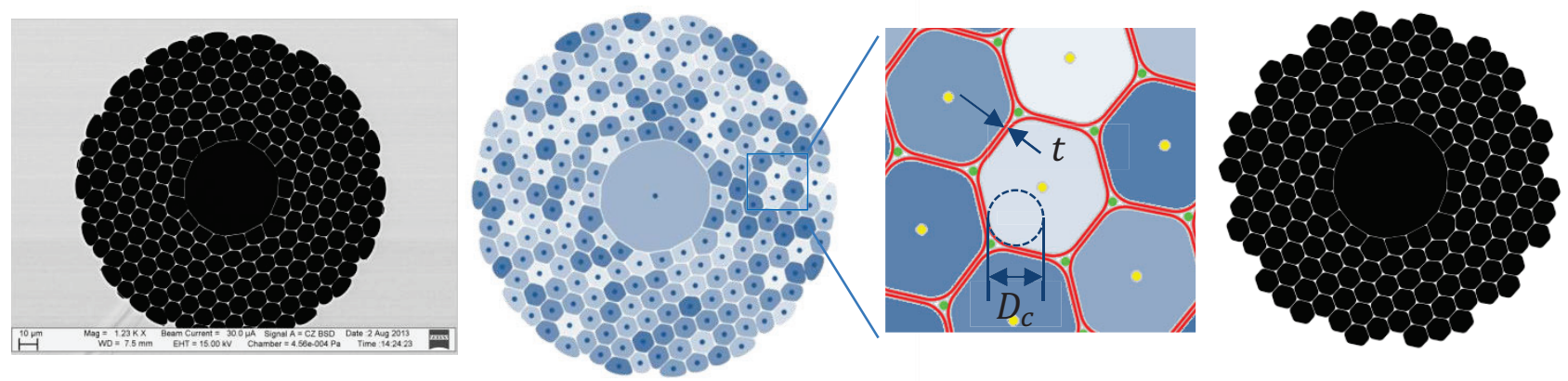

Fig. 1: Procedure to reconstruct the fiber geometry from an SEMs (left). The central position of all holes and nodes is automatically detected (center) and used to recreate the fiber structure (right) using two free parameters, $t$ and $D_{c}$. 
First we filter and convert the grayscale image into a binary black and white one. At this stage, each air hole can be identified and its center of mass accurately located by using simple image processing routines (e.g. in MATLAB). We then apply a dilation to each air hole with a structural element appropriately chosen so that all the air holes are merged together. This leaves the interstitials nodes as isolated objects, the center of mass of which can be also accurately located. Once the position of the center of each air hole and interstitial node is found, a hexagon (or pentagon) is built around each air hole by finding the six (or five) closest nodes to its center. Each edge of the hexagon (pentagon) is then moved closer to the hole's center by a distance corresponding to half the desired strut thickness $t$, which in this model is a free parameter. The second free parameter is $D_{c}$ the fillet diameter used to round the corners of the resulting hole (Fig.1). A good estimate of these two parameters can be obtained from averaging measurements on highly magnified portions of the cross section, adjusted by the estimated coating thickness. Alternatively a mass-conservation model predicting the evolution of second-stage preforms into fibers can be used [5]. The freedom to create struts of arbitrary thickness allows one to generate geometries better matching the actual fiber, avoiding the limitation posed by the finite SEM resolution. Note that since no nodes lay beyond the outermost ring of air holes, this final ring is not reproduced in the final geometry. Nonetheless, its omission has very little effect on the fiber's confinement loss which remains negligible, while scattering and absorption loss are largely unaffected. To make the generated structure even more similar to that of the real fiber, we apply mass conservation to each silica strut. Struts with lengths corresponding to the average strut length are made of thickness $t$, while longer and shorter are made thinner and thicker, respectively, by imposing their thickness to be inversely proportional to their length. This is particularly important for the struts on the core boundary, the thickness of which determines whether or not surface modes are supported within the photonic bandgap [6].

\section{Results and discussion}

We have used the method described above to model the optical properties of several fabricated fiber samples. Figure 2 shows simulation results for two fibers drawn from similar preforms, but scaled to operate around $1.55 \mu \mathrm{m}$ and $2.0 \mu \mathrm{m}$ respectively.
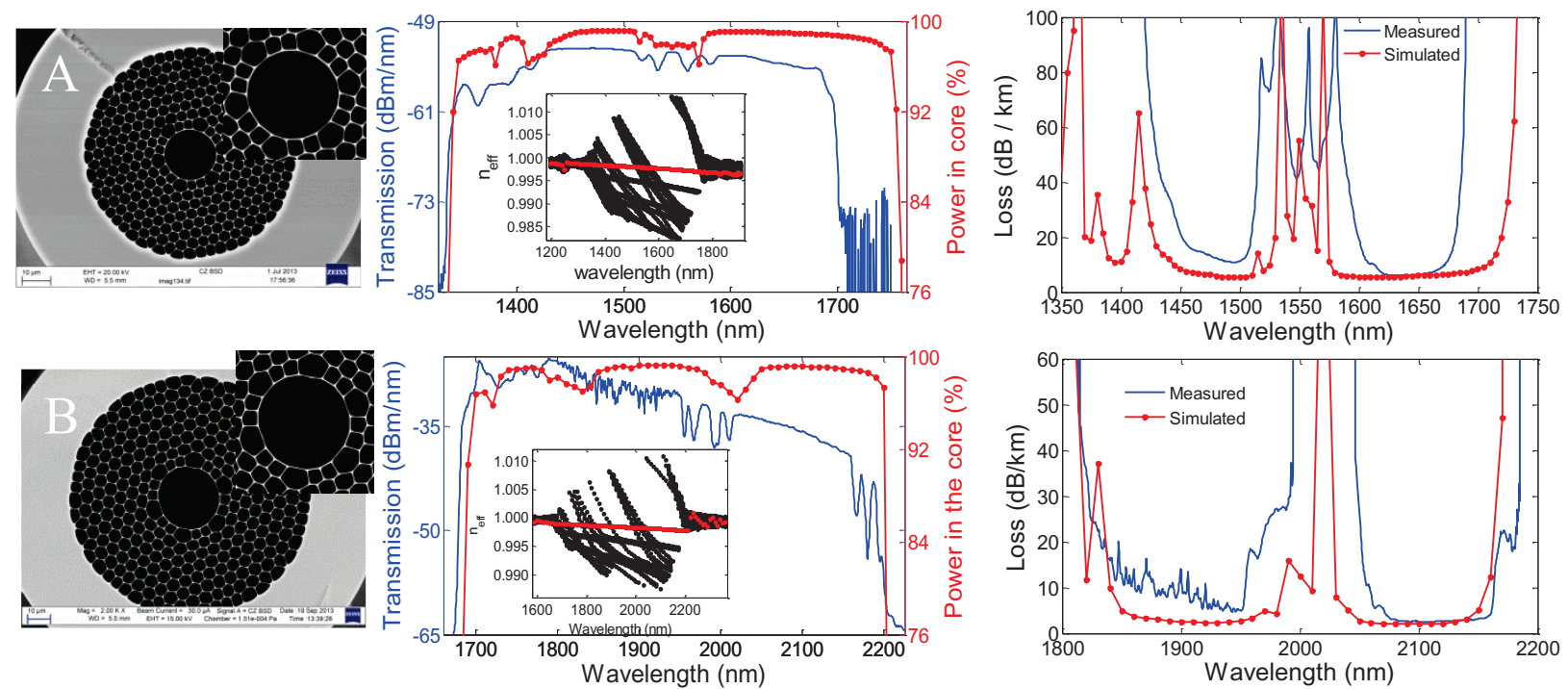

Fig. 2: Comparison between simulation results and measurements for a fiber operating: (A) around 1.55 um and (B) near 2 um. The inset in the central figures is a modal effective index map showing the multiple surface modes crossing the air guide mode (red curve). The simulated loss in the figures on the right is calculated as the sum of scattering and confinement loss contributions.

Fiber A, drawn to operate near $1.55 \mu \mathrm{m}$, has average hole-to-hole spacing, strut thickness and average fillet diameter of $\Lambda=4.02 \mu \mathrm{m}, t=100 \mathrm{~nm}$ and $D_{c}=0.5 \Lambda$, respectively. Fiber B made to operate around $2 \mu \mathrm{m}$ has average parameters $\Lambda=5.3 \mu \mathrm{m}, t=120 \mathrm{~nm}$ and $D_{c}=0.45 \Lambda$. As can be observed from the plots of Fig 2 , the simulated and measured photonic bandgaps are in good agreement. More importantly, the surface modes positions within the bandgap match pretty accurately the measurements, with a large group of them guided at center bandgap. This arises from the fact that the preforms employed had a thin core tube, thus making the strut edge on the core boundary thicker than those in the cladding [6]. The wider surface mode affected region observed in real fibers could arise from small longitudinal variations of the structure.

At these wavelengths the loss is dominated by scattering from surface roughness, since the incorporation of seven rings of air holes outside the core limits leakage loss to negligible values [7]. Simulations on numerous fibers 
revealed that the scattering loss can be inferred with excellent fidelity from the simulated normalized interface field intensity, given by:

$$
F=\left(\frac{\varepsilon_{0}}{\mu_{0}}\right)^{\frac{1}{2}} \frac{\int_{\text {hole perimeters }}|E|^{2} d s}{\iint_{\text {cross-section }} \boldsymbol{E} \times \boldsymbol{H}^{*} d A} .
$$

where, $E$ and $H$ are the electric and magnetic fields respectively. For our fibers, we found that $F=0.0116 \mu m^{-1}$ around $1.5 \mu \mathrm{m}$ corresponds to a loss value of $3.5 \mathrm{~dB} / \mathrm{km}$. Additionally, scattering loss for fibers operating at different wavelengths follows the well-known $\lambda^{-3}$ wavelength dependence [7]. Using this simple scaling, the simulated minimum loss obtained for several samples with photonic bandgaps centered at different wavelengths has shown remarkable agreement with cutback measurements, as can be seen in Fig. 2. This is to the best of our knowledge the first time simulated loss values for fabricated fibers have shown good agreement with measurements. The small discrepancies in bandwidth and loss between simulations and experiment could indicate some residual inaccuracies when generating the profile, or they may result from longitudinal variations along the fiber.

We also performed finite element calculations for the fiber in Fig 1, which was drawn from a preform stacked without a core tube and designed to operate in the mid-infrared near $3.4 \mu \mathrm{m}$ (with $\Lambda=9.3 \mu \mathrm{m}, t \approx 300 \mathrm{~nm}$ and $D_{c}=0.45$ )[8]. Here, material absorption rather than scattering dominates the loss, as shown in Fig. 3.

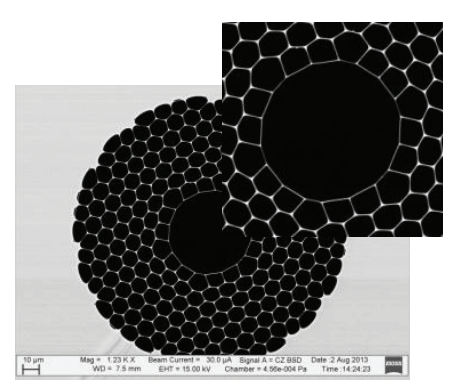

Fig. 3: Transmission and loss measurement versus simulated power in the core and loss for a fabricated mid-IR HC-PBGF. Simulations with and without the glass absorption clearly indicate the dominant source of loss at these wavelengths.

Our simulation used the wavelength dependent absorption of dry silica (Suprasil F300) extracted from the data of Humbach et al. [9]. Even in this case the simulated loss agrees well with the experimental data, particularly at longer wavelengths, confirming that despite the low modal overlap with glass $(<0.2 \%)$ the loss in this region is dominated by the glass absorption [8]. We note that as a result of the thin core surround no surface modes appear near the center of the photonic bandgap, neither in the simulation nor in the real fiber. The oscillations seen in the loss curve are due to modal beating (3100-3300nm) and gas absorption (3300-3600nm) [8].

\section{Conclusion}

We have presented a novel, accurate method to reproduce the structural profiles of HC-PBGFs from SEM images of their cross-section. Our method overcomes the previous issue of insufficient image resolution. Finite element method simulations performed on various fabricated fibers show for the first time that the loss and surface mode positions in fabricated HC-PBGFs can be accurately modeled. Our simulations indicate that distortions near the core play an important role in determining the scattering loss of the fiber. The method outlined here shall therefore serve as a valuable verification tool in HC-PBGF design, and can allow the study of how small structural changes around the core impact the loss and bandwidth of these fibers.

\section{Acknowledgements}

This work was supported by the EU 7th Framework Programme under grant agreement 258033(MODE-GAP) and by the UK EPSRC through grants EP/I01196X/1 (HYPERHIGHWAY) and EP/H02607X/1(IMRC).

\section{References}

[1] Poletti et al, Nature Photonics 7, 279-284 (2013).

[2] Li et al, J. Lightwave Technol. 25, 2463-2468 (2007).

[3] Poletti et al, Proceedings of OFC 2006, paper OFC2.

[4] Aghaie et al, J. Lightwave Technol. 31, 1015-1022 (2013).

[5] Numkam Fokoua et al, Optics Letters 2013 Vol.38(9) pp.1382-

1384

[6] Poletti et al, ECOC 2013 London 22-26 Sep 2013 Tu.3A.4
[7] Numkam Fokoua et al, Optics Express 2012 Vol.20(19) pp.20980-20991.

[8] Wheeler et al, submmited to Optics Letters

[9] Humbach et al, Journal of Non-Crystalline Solids, vol. 203, pp. 19-26, 1996. 